Homology, Homotopy and Applications, vol.8(1), 2006, pp.169-186

\title{
UNSTABLE SPLITTING OF $V(1) \wedge V(1)$ AND ITS APPLICATIONS
}

TAKAHISA SHIINA

(communicated by Donald M. Davis)

Abstract

Let $P^{n}(p)$ be an $n$-dimensional $\bmod p$ Moore space and $V^{n}$ be the mapping cone of an Adams map $A: P^{n-1}(p) \rightarrow$ $P^{n-2 p+1}(p)$. This paper gives an unstable splitting of $V^{m} \wedge V^{n}$ for a prime $p \geqslant 5$. The proof is based on explicit calculations of $\left[V^{n+2 p-1}, V^{n}\right]$. As an application, we define a Samelson product on $\left[V^{*}, \Omega X\right]$ and prove that it satisfies anticommutativity and the Jacobi identity.

\section{Introduction}

\subsection{Motivation}

Cohen, Moore, and Neisendorfer proved some properties of mod $p$ Moore spaces in [2]. For example, they proved the $\bmod p$ Hurewicz theorem and the product decomposition of the loop space of Moore space. This product decomposition yields a map $\pi_{n}: \Omega^{2} S^{2 n+3} \rightarrow S^{2 n+1}$ and this map plays a major role in [5].

Gray introduced the concept of EHP spectrum in [5]. An EHP spectrum of period $2 d$ is a spectrum $X=\left\{X_{n}\right\}$, where each $X_{n}$ is localized at an odd prime $p$, together with fiber sequences

$$
\begin{aligned}
& \cdots \stackrel{P}{\longrightarrow} X_{2 n-1} \stackrel{E}{\longrightarrow} \Omega X_{2 n} \stackrel{H^{\prime}}{\longrightarrow} \Omega X_{2 k n-1}, \\
& \cdots \stackrel{P}{\longrightarrow} X_{2 n} \stackrel{E}{\longrightarrow} \Omega X_{2 n+1} \stackrel{H}{\longrightarrow} \Omega X_{2 k n+2 d+1} .
\end{aligned}
$$

Gray also defined that an EHP spectrum has property CMN if there is a map

$$
\pi_{n}: \Omega^{2} X_{2 n+2 d+1} \longrightarrow X_{2 n-1},
$$

making the diagram

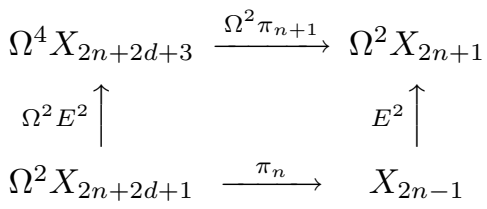

commutative and

$$
\Omega \pi_{n k} \simeq H^{\prime} \circ \Omega P: \Omega^{3} X_{2 k n+2 d+1} \longrightarrow \Omega X_{2 k n-1} .
$$

Received June 22, 2005, revised October 14, 2005; published on November 29, 2005.

2000 Mathematics Subject Classification: 55P15, 55Q15.

Key words and phrases: $V(1)$, Samelson product.

Copyright (C) 2005, International Press. Permission to copy for private use granted. 
The Smith-Toda spectra satisfy that if there exists an EHP spectrum representing $V(m)$, then $V(m+1)$ exists. Moreover, if an EHP spectrum representing $V(m)$ has property CMN, then we can construct a derived EHP spectrum and it represents $V(m+1)$.

The sphere spectrum $\hat{S}$,

$$
\hat{S}_{n}= \begin{cases}S^{n}, & n=2 m+1, \\ J_{p-1} S^{2 n}, & n=2 m,\end{cases}
$$

is an EHP spectrum of period 0 and $k=p$. It represents $V(-1)$ and has property CMN by a map $\pi_{n}: \Omega^{2} S^{2 n+3} \rightarrow S^{2 n+1}$ constructed by Cohen, Moore, and Neisendorfer in [2]. Gray states in [5, Section 6] that the CMN map on $V(0)$ might be constructed in the same way as the CMN map $\pi_{n}$ on $V(-1)$.

In $[\mathbf{2}]$, the mod $p$ Hurewicz theorem and the Samelson product on $\left[P^{*}(p), \Omega X\right]$ are the most important tools used to construct the product decomposition of the loop space of a Moore space. So, such tools might be needed to construct the CMN map on $V(0)$, and preparing such tools is a main object of this paper.

\subsection{Main theorems}

Let $p$ be a prime greater than or equal to $5, P^{n}(p)=S^{n-1} \cup_{p} e^{n}$, i.e. the mapping cone of a degree $p$ map, $A: P^{n+2 p-2}(p) \rightarrow P^{n}(p)$ be an Adams map, and $V^{n}=$ $P^{n-2 p+1}(p) \cup_{A} C P^{n-1}(p)$, i.e. the mapping cone of an Adams map. In this paper, we assume that all spaces are localized at $p$ and all (co)homologies are mod $p$ (co)homology.

In Section 3, we prove the following theorem, which refers to the Hurewicz map $\left[V^{n}, X\right] \rightarrow H_{n}(X)$.

Theorem 1.1. Assume that $X$ is an $r$-connected space. If $x \in H_{n}(X), n \leqslant \min \{2 r$, $\left.r+2 p^{2}-2 p-3\right\}$ and $\mathcal{P}_{*}^{1}(x)=0$, then there exists $f \in\left[V^{n}, X\right]$ such that $\varphi(f)=x$. Moreover, if $\mathcal{P}_{*}^{1}: H_{n+1}(X) \rightarrow H_{n-2 p+3}(X)$ is surjective, then $f$ is unique.

In Section 4 , we calculate the homotopy set $\left[V^{6 p+2}, V^{4 p+3}\right]$.

Lemma 1.2. $\left[V^{6 p+2}, V^{4 p+3}\right]=0$.

We remark that it fails in the case of $p=3$. This homotopy set is calculated in order to show the unstable splitting of $V(1) \wedge V(1)$.

Theorem 1.3. Assume that $m, n \geqslant 2 p+3$ and $m+n \geqslant 6 p+3$. Then there is a space $M$ and a homotopy equivalence

$$
V^{m} \wedge V^{n} \longrightarrow V^{m+n} \vee V^{m+n-2 p} \vee M .
$$

This theorem is proved in Section 5. This splitting defines the Samelson product on $\left[V^{*}, \Omega X\right]$. The next problem is whether the Samelson product defined by using this splitting provides a Lie algebra structure on $\left[V^{*}, \Omega X\right]$ or not. The answer is the following theorem which is proved in Section 6. 
Theorem 1.4. For $f: V^{m} \rightarrow \Omega X$ and $g: V^{n} \rightarrow \Omega X$, the Samelson product

$$
[f, g]: V^{m+n} \longrightarrow V^{m} \wedge V^{n} \stackrel{f \wedge g}{\longrightarrow} \Omega X \wedge \Omega X \stackrel{\langle,\rangle}{\longrightarrow} \Omega X
$$

satisfies the following properties:

1. $\left[f+f^{\prime}, g\right]=[f, g]+\left[f^{\prime}, g\right],\left[f, g+g^{\prime}\right]=[f, g]+\left[f, g^{\prime}\right]$ (bilinear),

2. $[f, g]=-(-1)^{m n}[g, f]$ (anticommutative) if $m+n \geqslant 8 p+2$,

3. $(-1)^{m k}[f,[g, h]]+(-1)^{m n}[g,[h, f]]+(-1)^{n k}[h,[f, g]]$ (Jacobi identity) if $m+$ $n+k \geqslant 12 p+2$, where $h: V^{k} \rightarrow \Omega X$,

4. $h_{*}[f, g]=\left[h_{*} f, h_{*} g\right]$ for an $H$-map $h: \Omega X \rightarrow \Omega Y$,

5. $\varphi([f, g])=[\varphi(f), \varphi(g)]$ where $\varphi:\left[V^{i}, \Omega X\right] \rightarrow H_{i}(\Omega X)$ is the Hurewicz map.

\section{Properties}

In this section, we recall some results about homotopy sets of Moore spaces proved in [2], and the relative James construction introduced in [4].

For $\bmod p$ Moore space $P^{n}(p)$, let $v_{n}$ and $u_{n-1}$ be generators of $H_{*}\left(P^{n}(p)\right)$ in degree $n$ and $n-1$, respectively, with $\beta\left(v_{n}\right)=u_{n-1}$ where $\beta$ is the Bockstein homomorphism. The inclusion of the bottom cell is denoted by $i: S^{n-1} \rightarrow P^{n}(p)$. We have a cofibration $S^{n-1} \stackrel{i}{\rightarrow} P^{n}(p) \stackrel{q}{\rightarrow} S^{n}$. Denote the identity map by $\iota: P^{n}(p) \rightarrow$ $P^{n}(p)$ and the composition $i q$ by $\delta: P^{n}(p) \rightarrow P^{n+1}(p)$. According to [3], the Adams map $A: P^{n+2 p-2}(p) \rightarrow P^{n}(p)$ exists for $n \geqslant 3$.

\subsection{Some homotopy sets}

The stable homotopy set $\operatorname{colim}_{k}\left[P^{n+k}(p), P^{n}(p)\right]$ is known for small values of $k$.

Proposition 2.1 (Toda $[\mathbf{9}]$ ).

$$
\lim _{n \rightarrow \infty}\left[P^{n+k}(p), P^{n}(p)\right] \cong \begin{cases}\mathbb{Z} / p \mathbb{Z}\{\delta\}, & k=-1, \\ \mathbb{Z} / p \mathbb{Z}\{\iota\}, & k=0, \\ \mathbb{Z} / p \mathbb{Z}\left\{\delta A^{r} \delta\right\}, & k=r q-2, \\ \mathbb{Z} / p \mathbb{Z}\left\{\delta A^{r}, A^{r} \delta\right\}, & k=r q-1, \\ \mathbb{Z} / p \mathbb{Z}\left\{A^{r}\right\}, & k=r q, \\ 0, & \text { otherwise, }\end{cases}
$$

where $q=2 p-2$ and $r<p$.

The $\bmod p$ homotopy groups of spheres are known as follows.

Proposition 2.2 (Toda [8]).

$$
\left[P^{2 n+1+i}(p), S^{2 n+1}\right] \cong \begin{cases}\mathbb{Z} / p \mathbb{Z}\{q\}, & i=0, \\ \mathbb{Z} / p \mathbb{Z}\{q A \delta\}, & i=2 p-3, \\ \mathbb{Z} / p \mathbb{Z}\{q A\}, & i=2 p-2, \\ \mathbb{Z} / p \mathbb{Z}\left\{q A^{2} \delta\right\}, & i=4 p-5, \\ \mathbb{Z} / p \mathbb{Z}\left\{q A^{2}\right\}, & i=4 p-4, \\ 0, & \text { otherwise for } i<6 p-8 .\end{cases}
$$


Let $S^{n}\{p\}$ be the homotopy fiber of $S^{n} \stackrel{p}{\rightarrow} S^{n}$. The mod $p$ homotopy groups of $S^{2 n+1}\{p\}$ are known by considering the fiber sequence

$$
\Omega S^{2 n+1} \stackrel{k}{\rightarrow} S^{2 n+1}\{p\} \stackrel{j}{\rightarrow} S^{2 n+1} \stackrel{p}{\rightarrow} S^{2 n+1} .
$$

\section{Corollary 2.3.}

$$
\left[P^{2 n+1+i}(p), S^{2 n+1}\{p\}\right] \cong \begin{cases}\mathbb{Z} / p \mathbb{Z}\{j E q\}, & i=-1, \\ \mathbb{Z} / p \mathbb{Z}\left\{q^{\prime}\right\}, & i=0, \\ \mathbb{Z} / p \mathbb{Z}\{j E q A \delta\}, & i=2 p-4, \\ \mathbb{Z} / p \mathbb{Z}\left\{j E q A,(q A \delta)^{\prime}\right\}, & i=2 p-3, \\ \mathbb{Z} / p \mathbb{Z}\left\{(q A)^{\prime}\right\}, & i=2 p-2, \\ \mathbb{Z} / p \mathbb{Z}\left\{j E q A^{2} \delta\right\}, & i=4 p-6, \\ \mathbb{Z} / p \mathbb{Z}\left\{j E q A^{2},\left(q A^{2} \delta\right)^{\prime}\right\}, & i=4 p-5, \\ \mathbb{Z} / p \mathbb{Z}\left\{\left(q A^{2} \delta\right)^{\prime}\right\}, & i=4 p-4, \\ 0, & \text { otherwise for } i<6 p-9,\end{cases}
$$

where $(-)^{\prime}$ is a lift

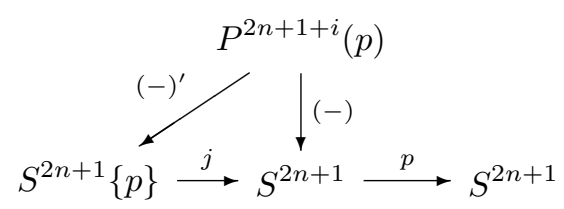

\subsection{Properties of Moore space}

The following theorem is frequently used in this paper.

Theorem $2.4(\bmod p$ Hurewicz theorem $[7])$. Assume that $\left[P^{i}(p), X\right]=0$ for all $2 \leqslant i \leqslant n$. Then the Hurewicz map

$$
\phi:\left[P^{i}(p), X\right] \longrightarrow H_{i}(X)
$$

defined by $\phi(f)=f_{*}\left(v_{i}\right)$ is an isomorphism for $i \leqslant \min \{2 n, n+2 p-3\}$ and surjective for $i=\min \{2 n, n+2 p-3\}+1$.

From this theorem, we have the splitting of the smash product of Moore spaces.

Theorem 2.5 (Cohen, Moore, and Neisendorfer [2]). Assume that $m+n \geqslant 6$. Then there is a homotopy equivalence

$$
P^{m+n}(p) \vee P^{m+n-1}(p) \longrightarrow P^{m}(p) \wedge P^{n}(p) .
$$

For $f: P^{m}(p) \rightarrow \Omega X$ and $g: P^{n}(p) \rightarrow \Omega X$, the Samelson product of $f$ and $g$, $[f, g]$, is the composition

$$
\begin{aligned}
{[f, g]: P^{m+n}(p) \longrightarrow P^{m+n}(p) \vee P^{m+n-1}(p) \stackrel{\simeq}{\longrightarrow} P^{m}(p) \wedge P^{n}(p) } \\
\quad \stackrel{f \wedge g}{\longrightarrow} \Omega X \wedge \Omega X \stackrel{\langle,\rangle}{\longrightarrow} \Omega X
\end{aligned}
$$

where $\langle$,$\rangle is the commutator map. This Samelson product gives a Lie algebra struc-$ ture on $\left[P^{*}(p), \Omega X\right]$. The algebra $H_{*}(\Omega X)$ is an associative, and it can be regarded as 
a Lie algebra, i.e. $[x, y]=x y-(-1)^{|x||y|} y x$ for $x, y \in H_{*}(\Omega X)$. Then the Hurewicz map is a Lie algebra homomorphism, i.e.

$$
\phi([f, g])=[\phi(f), \phi(g)] .
$$

The Freudenthal suspension $E \in\left[P^{n}(p), \Omega P^{n+1}(p)\right]$ and the composition $E \delta$ correspond to $\nu_{n} \in H_{n}\left(\Omega P^{n+1}(p)\right)$ and $\mu_{n-1} \in H_{n-1}\left(\Omega P^{n+1}(P)\right)$ under the Hurewicz map, respectively.

Theorem 2.6 ([2]). There exist homotopy equivalences

$$
S^{2 n+1}\{p\} \times \Omega \Sigma\left(\bigvee_{k=0}^{\infty} P^{4 n+2 k n+2}(p)\right) \stackrel{\simeq}{\longrightarrow} \Omega P^{2 n+2}(p)
$$

and

$$
S^{2 n-1} \times \prod_{k=1}^{\infty} S^{2 p^{k} n-1}\left\{p^{2}\right\} \times \Omega \Sigma\left(\bigvee_{n_{\alpha}} P^{n_{\alpha}}(p)\right) \stackrel{\simeq}{\longrightarrow} \Omega F^{2 n+1}\{p\}
$$

for all $n \geqslant 1$. Where $F^{2 n+1}\{p\}$ is the fiber of the pinch map $P^{2 n+1}(p) \stackrel{q}{\rightarrow} S^{2 n+1}$ and $n_{\alpha} \geqslant 4 n+1$

Denote $g_{k}=\overbrace{[E \delta,[\ldots,[E \delta,}^{k},[E, E]]]]$. Then the next diagram

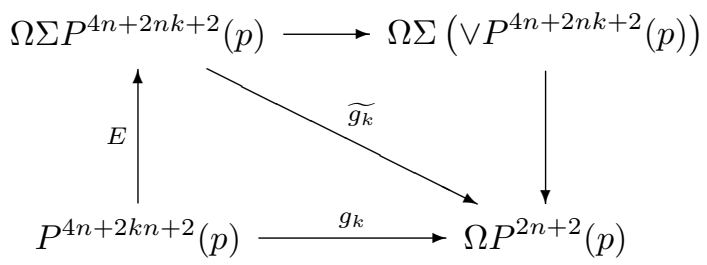

is commutative. Similarly, there is a map $g_{n_{\alpha}}: P^{n_{\alpha}}(p) \rightarrow \Omega P^{2 n+1}(p)$ such that the diagram

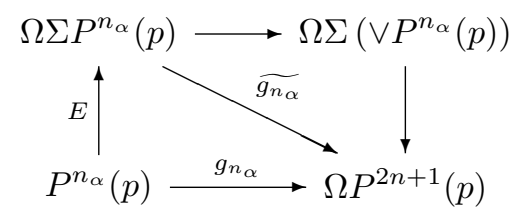

is commutative. This $g_{n_{\alpha}}$ is a Samelson product consisting of some $E$ and $E \delta$. For example, in low dimensions,

$$
\left.g_{\alpha}=[E, E \delta],[E,[E, E \delta]], \ldots,[\overbrace{E, \ldots,[E}^{p-2},[E, E \delta]]\right], \ldots,
$$

$n_{a}=4 n-1,6 n-1, \ldots, 2 n p-1, \ldots$, and so on.

\subsection{Relative James construction}

Let $B$ be a subspace of a space $X$. The relative James construction $(X, B)_{\infty}$ is the set of words in the James construction $X_{\infty}$ whose letters, except the first letter, 
are in $B$. If $(X, B)$ is an NDR then there is a weak homotopy equivalence

$$
f:(X, B)_{\infty} \longrightarrow G,
$$

where $G$ is the fiber of the pinch map $q: X \cup C B \rightarrow \Sigma B$. In addition, the diagram

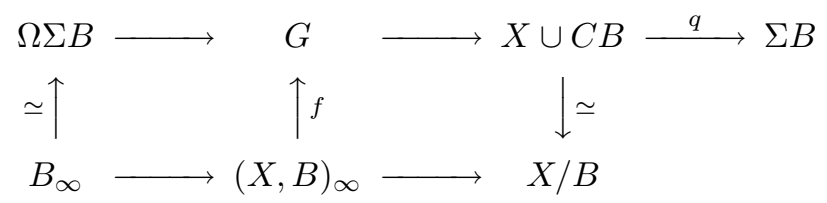

is commutative, where the left homotopy equivalence is James' and the right homotopy equivalence is given by the NDR structure of $(X, B)$.

For each $n$, we have a map called the Hopf invariant $H_{n}:(X, B)_{\infty} \rightarrow$ $\left(X \wedge B^{\wedge(n-1)}\right)_{\infty}$. Let

$$
\begin{aligned}
& N=\prod_{n \geqslant 1}\left(X \wedge B^{\wedge n}\right)_{\infty}=\underset{k}{\operatorname{hocolim}} \prod_{n=1}^{k}\left(X \wedge B^{\wedge n}\right), \\
& H_{(X, B)}=\prod H_{n}:(X, B)_{\infty} \rightarrow N,
\end{aligned}
$$

and $F_{(X, B)}$ be the homotopy fiber of $H_{(X, B)}$. By combining the following two exact sequences

$$
\begin{aligned}
& \cdots \longrightarrow\left[Y,(X, B)_{\infty}\right] \longrightarrow[Y, X \cup C B] \stackrel{q_{*}}{\longrightarrow}[Y, \Sigma B], \\
& \left.\cdots \longrightarrow\left[Y, F_{(X, B)}\right] \longrightarrow[X, B)_{\infty}\right] \stackrel{H_{(X, B) *}}{\longrightarrow}[Y, N],
\end{aligned}
$$

we obtain the following diagram:

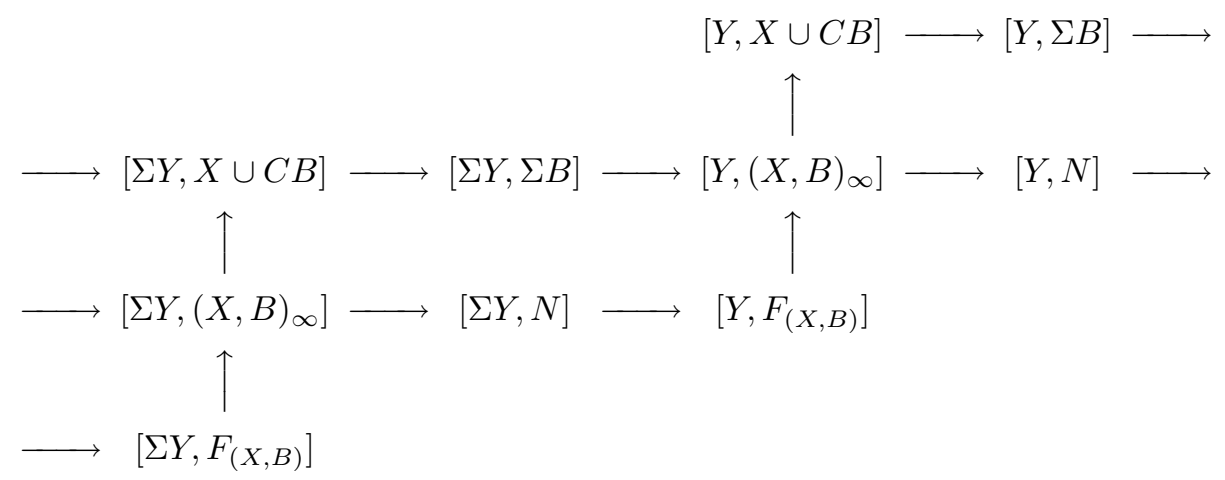

Since the composite of inclusion $X \stackrel{i}{\rightarrow}(X, B)_{\infty}$ and $H_{(X, B)}:(X, B)_{\infty} \rightarrow N$ is null homotopic, there is a lift $\psi: X \rightarrow F_{(X, B)}$ of $i$. Gray said in [4] that the diagram

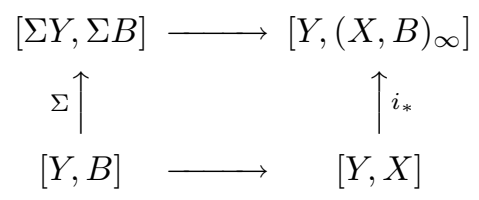

is commutative. 
We now consider the case of $Y=P^{n}(p)$. Referring to [4, Theorem 8.1], we obtain the following lemma.

Lemma 2.7. If

$$
\begin{array}{ll}
{\left[P^{i}(p), X\right]=0} & \text { for } i<m, \\
{\left[P^{i}(p), B\right]=0} & \text { for } i<n,
\end{array}
$$

then $\psi_{*}:\left[P^{i}(p), X\right] \rightarrow\left[P^{i}(p), F_{(X, B)}\right]$ is an isomorphism for $i \leqslant 2 m+2 n-3$.

Proof. From [4], we know

$$
\begin{aligned}
\widetilde{H}_{*}\left((X, B)_{\infty}\right) & \cong \widetilde{H}_{*}(X) \otimes H_{*}\left(B_{\infty}\right) \\
& \cong \widetilde{H}_{*}(X) \oplus\left(\widetilde{H}_{*}(X) \otimes \widetilde{H}_{*}(B)\right) \oplus\left(\widetilde{H}_{*}(X) \otimes \widetilde{H}_{*}(B)^{\otimes 2}\right) \oplus \cdots .
\end{aligned}
$$

By the definition of $N$,

$$
\begin{aligned}
H_{*}(N) & \cong \bigotimes_{n \geqslant 1} T\left(\widetilde{H}_{*}\left(X \wedge(B)^{\wedge n}\right)\right) \\
& \cong\left\{\mathbb{Z} / p \mathbb{Z} \oplus\left(\widetilde{H}_{*}(X) \otimes \widetilde{H}_{*}(B)\right) \oplus\left(\widetilde{H}_{*}(X) \otimes \widetilde{H}_{*}(B)\right)^{\otimes 2} \oplus \cdots\right\} \\
& \otimes\left\{\mathbb{Z} / p \mathbb{Z} \oplus\left(\widetilde{H}_{*}(X) \otimes \widetilde{H}_{*}(B)^{\otimes 2}\right) \oplus\left(\widetilde{H}_{*}(X) \otimes \widetilde{H}_{*}(B)^{\otimes 2}\right)^{\otimes 2} \oplus \cdots\right\} \\
& \otimes \cdots,
\end{aligned}
$$

where $T(-)$ means the tensor algebra. For $x \in \widetilde{H}_{m}(X)$ and $a \in \widetilde{H}_{n}(B),(x \otimes a)^{2} \in$ $\widetilde{H}_{2 m+2 n}(N)$ is the lowest degree element which is not included in the image of $H_{(X, B) *}$. The Serre spectral sequence of the fibration

$$
F_{(X, B)} \rightarrow(X, B)_{\infty} \stackrel{H_{(X, B)}}{\longrightarrow} N
$$

implies that $\psi_{*}: H_{i}(X) \rightarrow H_{i}\left(F_{(X, B)}\right)$ is an isomorphism for $i \leqslant 2 m+2 n-2$. Hence, $\psi_{*}:\left[P^{i}(p), X\right] \rightarrow\left[P^{i}(p), F_{(X, B)}\right]$ is an isomorphism for $i \leqslant 2 m+2 n-3$.

We close this section after stating the following two classical theorems.

Theorem 2.8 (Freudenthal suspension theorem). Let $X$ be a $C W$ complex and $Y$ be a r-connected space. Then the suspension map

$$
\Sigma:[X, Y] \longrightarrow[\Sigma X, \Sigma Y]
$$

is bijective if $\operatorname{dim} X \leqslant 2 r$.

Theorem 2.9 (Hilton-Milnor theorem).

$$
\Omega \Sigma(X \vee Y) \simeq \Omega \Sigma X \times \Omega \Sigma \bigvee_{k \geqslant 0}\left(Y \wedge X^{\wedge k}\right)
$$


Homology, Homotopy and Applications, vol. 8(1), 2006

\section{The Hurewicz map}

Let $a_{n}, b_{n-1}, c_{n-2 p+1}$ and $d_{n-2 p}$ be the basis of $H_{*}\left(V^{n}\right)$ in degree $n, n-1$, $n-2 p+1$ and $n-2 p$ respectively. The Hurewicz map

$$
\varphi:\left[V^{n}, X\right] \longrightarrow H_{n}(X)
$$

is defined by $\varphi(f)=f_{*}\left(a_{n}\right)$. Theorem 1.1 is used to prove the unstable splitting of $V^{m} \wedge V^{n}$ and the Samelson product, and Theorem 1.1 follows from the next proposition.

Proposition 3.1. Let $E$ be a spectrum of the fiber of $\mathcal{P}^{1}: H \mathbb{Z} / p \mathbb{Z} \rightarrow \Sigma^{2 p-2} H \mathbb{Z} / p \mathbb{Z}$ where $H \mathbb{Z} / p \mathbb{Z}$ is the Eilenberg-MacLane spectrum of $\mathbb{Z} / p \mathbb{Z}$. Assume that $X$ is a $p$ local and $r$-connected space. Then the Hurewicz map $\varphi^{\prime}:\left[V^{i}, X\right] \rightarrow E_{i}(X)$ is bijective for $i \leqslant \min \left\{2 r, r+2 p^{2}-2 p-3\right\}$ and surjective for $i=\min \left\{2 r, r+2 p^{2}-2 p-\right.$ $3\}+1$.

Proof of Theorem 1.1. It is proved by considering the diagram

$$
H_{n+1}(X) \stackrel{\mathcal{P}_{*}^{1}}{\longrightarrow} H_{n-2 p+3}(X) \stackrel{j_{*}}{\longrightarrow} E_{n}(X) \underset{\varphi^{\prime}}{\stackrel{i_{*}}{\longrightarrow}} \overbrace{\left[V^{n}, X\right]} H_{n}(X) \stackrel{\mathcal{P}_{*}^{1}}{\longrightarrow} H_{n-2 p+2}(X)
$$

where the upper sequence is exact.

We prove Proposition 3.1 in the rest of this section.

We first recall the Steenrod algebra $\mathcal{A}^{*}$ and its dual $\mathcal{A}_{*}$. We know that

$$
\begin{aligned}
& \mathcal{A}^{*} \cong H^{*}(H \mathbb{Z} / p \mathbb{Z}), \\
& \mathcal{A}_{*} \cong H_{*}(H \mathbb{Z} / p \mathbb{Z}) .
\end{aligned}
$$

By Milnor [6], the algebra $\mathcal{A}_{*}$ is the tensor product of the exterior algebra generated by $\tau_{0}, \tau_{1}, \tau_{2}, \ldots$, and the polynomial algebra generated by $\xi_{1}, \xi_{2}, \ldots$, i.e.

$$
\mathcal{A}_{*} \cong E\left(\tau_{0}, \tau_{1}, \tau_{2}, \ldots\right) \otimes \mathbb{Z} / p \mathbb{Z}\left[\xi_{1}, \xi_{2}, \ldots\right],
$$

where $\operatorname{deg} \tau_{k}=2 p^{k}-1$ and $\operatorname{deg} \xi_{k}=2 p^{k}-2$. The element $\tau_{k}$ is the dual of $\mathcal{P}^{p^{k}} \mathcal{P}^{p^{k-1}} \ldots \mathcal{P}^{p} \mathcal{P}^{1} \beta$ and $\xi_{k}$ is the dual of $\mathcal{P}^{p^{k}} \mathcal{P}^{p^{k-1}} \cdots \mathcal{P}^{p} \mathcal{P}^{1}$. The algebra $\mathcal{A}_{*}$ is also a Hopf algebra by the coproduct

$$
\begin{aligned}
& \psi\left(\xi_{k}\right)=\sum_{i=0}^{k} \xi_{k-i}^{p^{i}} \otimes \xi_{i}, \\
& \psi\left(\tau_{k}\right)=\tau_{k} \otimes 1+\sum_{i=0}^{k} \xi_{k-i}^{p^{i}} \otimes \tau_{i} .
\end{aligned}
$$

Since $E$ is the spectrum of the fiber of $\mathcal{P}^{1}$, we obtain the fiber sequence

$$
\Sigma^{-1} H \mathbb{Z} / p \mathbb{Z} \stackrel{\mathcal{P}^{1}}{\longrightarrow} \Sigma^{2 p-3} H \mathbb{Z} / p \mathbb{Z} \stackrel{j}{\rightarrow} E \stackrel{i}{\rightarrow} H \mathbb{Z} / p \mathbb{Z} \stackrel{\mathcal{P}^{1}}{\longrightarrow} \Sigma^{2 p-2} H \mathbb{Z} / p \mathbb{Z} .
$$


Then the sequence

$$
\begin{aligned}
H_{i+1}(H \mathbb{Z} / p \mathbb{Z}) & \stackrel{\left(\mathcal{P}^{1}\right)_{*}}{\longrightarrow} H_{i-2 p+3}(H \mathbb{Z} / p \mathbb{Z}) \\
& \stackrel{j_{*}}{\longrightarrow} H_{i}(E) \stackrel{i_{*}}{\longrightarrow} H_{i}(H \mathbb{Z} / p \mathbb{Z}) \stackrel{\left(\mathcal{P}^{1}\right)_{*}}{\longrightarrow} H_{i-2 p+2}(H \mathbb{Z} / p \mathbb{Z})
\end{aligned}
$$

is exact. We calculate $H_{i}(E)$ for small $i$ from this exact sequence.

By reason of dimension, $\left(\mathcal{P}^{1}\right)_{*}(1)=0$ and $\left(\mathcal{P}^{1}\right)_{*}\left(\tau_{0}\right)=0$. From the definition of $\tau_{0}, \tau_{1}$ and $\xi_{1}$, we obtain that

$$
\begin{aligned}
\left(\mathcal{P}^{1}\right)_{*}\left(\tau_{1}\right) & =0 \\
\left(\mathcal{P}^{1}\right)_{*}\left(\xi_{1}^{k}\right) & =k \xi_{1}^{k-1}, \\
\left(\mathcal{P}^{1}\right)_{*}\left(\tau_{0}^{i} \tau_{1}^{j} \xi_{1}^{k}\right) & =k \tau_{0}^{i} \tau_{1}^{j} \xi_{1}^{k-1},
\end{aligned}
$$

where $i, j=0$ or 1 . The kernel of $\left(\mathcal{P}^{1}\right)_{*}$ is generated by $1, \tau_{0}, \tau_{1}, \tau_{0} \tau_{1}$ and $\xi_{1}^{p}$ in low dimensions. Since $\left(\mathcal{P}^{1}\right)_{*}\left(\xi_{1}^{p}\right)=p \xi_{1}^{p-1}=0, \xi_{1}^{p-1}$ is not included in image of $\left(\mathcal{P}^{1}\right)_{*}$. Therefore,

$$
H_{*}(E) \ni 1, \tau_{0}, \tau_{1}, \tau_{0} \tau_{1}, \widetilde{\xi_{1}^{p-1}}, \xi_{1}^{p}, \ldots
$$

where $\operatorname{deg} \widetilde{\xi_{1}^{p-1}}=2 p^{2}-2 p-1$. The homology operations $\beta$ and $\mathcal{P}_{*}^{1}$ on $\tau_{0}, \tau_{1}$ and $\tau_{0} \tau_{1}$ are as follows:

$$
\begin{aligned}
\beta\left(\tau_{0}\right) & =1, \\
\mathcal{P}_{*}^{1}\left(\tau_{1}\right) & =\tau_{0}, \\
\beta\left(\tau_{0} \tau_{1}\right) & =\tau_{1},
\end{aligned}
$$

Hence, for low dimensions, $H_{*}(E)$ is as follows:

$$
1 \stackrel{\beta}{-} \tau_{0} \stackrel{\mathcal{P}_{*}^{1}}{-} \tau_{1} \frac{\beta}{-} \tau_{0} \tau_{1} \quad \widetilde{\xi_{1}^{p-1}} \quad \xi_{1}^{p}
$$

with operations 0 on the latter classes.

Proof of Proposition 3.1. The Hurewicz map $\varphi^{\prime}:\left[V^{i}, X\right] \longrightarrow E_{i}(X)$ is obtained by the following sequence:

$$
\begin{aligned}
{\left[V^{i}, X\right] \stackrel{\Sigma^{\infty}}{\longrightarrow} } & {\left[\Sigma^{\infty} V^{i}, \Sigma^{\infty} X\right] \cong\left[\Sigma^{\infty} S^{i} \wedge \Sigma^{\infty} V^{0}, \Sigma^{\infty} X\right] } \\
& \cong\left[\Sigma^{\infty} S^{i}, \Sigma^{\infty} V^{2 p} \wedge \Sigma^{\infty} X\right] \stackrel{(\alpha \wedge 1)_{*}}{\longrightarrow}\left[\Sigma^{\infty} S^{i}, E \wedge \Sigma^{\infty} X\right]=E_{i}(X),
\end{aligned}
$$

where $\alpha$ is a generator of

$$
E^{0}\left(\Sigma^{\infty}\right)=\left[\Sigma^{\infty} V^{2 p}, E\right] \cong \mathbb{Z} / p \mathbb{Z} .
$$

This isomorphism is computed from the exact sequence

$$
\begin{aligned}
H^{j-1}\left(\Sigma^{\infty} V^{2 p}\right) & \stackrel{\mathcal{P}^{1}}{\longrightarrow} H^{j+2 p-3}\left(\Sigma^{\infty} V^{2 p}\right) \\
& \longrightarrow E^{j}\left(\Sigma^{\infty} V^{2 p}\right) \longrightarrow H^{j}\left(\Sigma^{\infty} V^{2 p}\right) \stackrel{\mathcal{P}^{1}}{\longrightarrow} H^{j+2 p-2}\left(\Sigma^{\infty} V^{2 p}\right),
\end{aligned}
$$


and

$$
\begin{aligned}
& \begin{array}{llll}
0 & 2 p-1 \quad 2 p
\end{array} \\
& H^{*}\left(\Sigma^{\infty} V^{2 p}\right)=\circ \stackrel{\beta}{-} \circ \mathcal{P}^{1} \circ \stackrel{\beta}{-} \circ
\end{aligned}
$$

where the integers above a node indicate the grading. So, $\alpha$ induces an isomorphism from $H_{i}\left(\Sigma^{\infty} V^{2 p}\right)$ to $H_{i}(E)$ for $i \leqslant 2 p^{2}-2 p-2$. Hence,

$$
(\alpha \wedge 1)_{*}:\left[\Sigma^{\infty} S^{i}, \Sigma^{\infty} V^{2 p} \wedge \Sigma^{\infty} X\right] \longrightarrow\left[\Sigma^{\infty} S^{i}, E \wedge \Sigma^{\infty} X\right]
$$

is an isomorphism for $i \leqslant r+2 p^{2}-2 p-3$ for $r$-connected space $X$. And since $X$ is $r$-connected,

$$
\Sigma^{\infty}:\left[V^{i}, X\right] \longrightarrow\left[\Sigma^{\infty} V^{i}, \Sigma^{\infty} X\right]
$$

is an isomorphism for $i \leqslant 2 r$. This completes the proof of the proposition.

\section{Calculation of $\left[V^{6 p+2}, V^{4 p+3}\right]$}

In this section, we prove $\left[V^{6 p+2}, V^{4 p+3}\right]=0$. From the cofiber sequence

$$
P^{6 p+1}(p) \stackrel{A}{\longrightarrow} P^{4 p+3}(p) \stackrel{i_{1}}{\longrightarrow} V^{6 p+2} \stackrel{q_{1}}{\longrightarrow} P^{6 p+2}(p) \stackrel{A}{\longrightarrow} P^{4 p+4}(p),
$$

we obtain an exact sequence of homotopy sets

$$
\left[P^{6 p+2}(p), V^{4 p+3}\right] \stackrel{q_{1}^{*}}{\longrightarrow}\left[V^{6 p+2}, V^{4 p+3}\right] \stackrel{i_{1}^{*}}{\longrightarrow}\left[P^{4 p+3}(p), V^{4 p+3}\right] .
$$

It is enough to show that

$$
\left[P^{6 p+2}(p), V^{4 p+3}\right]=0 \quad \text { and } \quad\left[P^{4 p+3}(p), V^{4 p+3}\right]=0 .
$$

They can be calculated by applying the relative James construction introduced in Section 2.3.

Let $X=P^{2 p+4}(p) \cup_{A} P^{4 p+2}(p) \times I$, the mapping cylinder of the Adams map $A: P^{4 p+2}(p) \rightarrow P^{2 p+4}(p)$, and $B=P^{4 p+2}(p) \times\{1\}$. Then, $X \simeq P^{2 p+4}(p)$, $B=P^{4 p+2}(p)$ and $X / B=V^{4 p+3}$.

By the definition,

$$
\begin{aligned}
N & =\prod_{k \geqslant 1}\left(X \wedge B^{\wedge k}\right)_{\infty}, \\
H_{(X, B)} & =\prod_{k} H_{k}(X, B)_{\infty} \rightarrow N,
\end{aligned}
$$

and $F_{(X, B)}$ is the homotopy fiber of $H_{(X, B)}$.

There is a map $\psi: X=P^{2 p+4}(p) \rightarrow F_{(X, B)}$. Since $\left[P^{i}(p), X\right]=0$ for $i<2 p+3$ and $\left[P^{i}(p), B\right]=0$ for $i<4 p+1$, from Lemma 2.7, the induced map

$$
\psi_{*}:\left[P^{i}(p), P^{2 p+4}(p)\right] \longrightarrow\left[P^{i}(p), F_{(X, B)}\right]
$$

is bijective for $i \leqslant 2(2 p+3)+2(4 p+1)-3=12 p+5$. In particular,

$$
\begin{aligned}
& {\left[P^{6 p+2}(p), P^{2 p+4}(p)\right] \longrightarrow\left[P^{6 p+2}(p), F_{(X, B)}\right],} \\
& {\left[P^{4 p+3}(p), P^{2 p+4}(p)\right] \longrightarrow\left[P^{4 p+3}(p), F_{(X, B)}\right]}
\end{aligned}
$$

are bijective. 
Homology, Homotopy and Applications, vol. 8(1), 2006

Now, in order to calculate $\left[P^{6 p+2}(p), V^{4 p+3}\right]$ and $\left[P^{4 p+3}(p), V^{4 p+3}\right]$, we apply the above data to relative James construction, and obtain the following diagram:

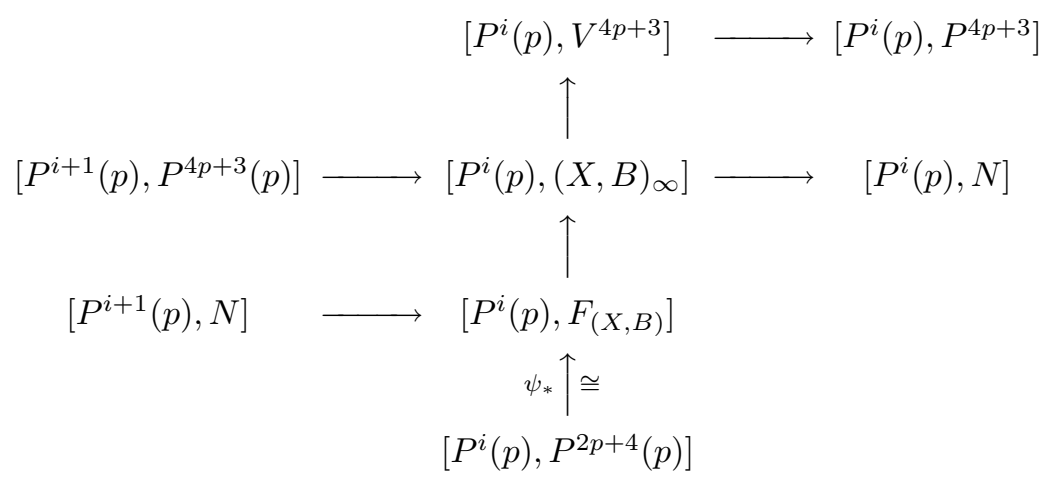

(i) $i=4 p+3$. By the definition of $N$,

$$
\left[P^{i}(p), N\right] \cong \prod_{k \geqslant 1}\left[P^{i}(p), \Omega \Sigma\left(P^{2 p+4}(p) \wedge\left(P^{4 p+2}(p)\right)^{\wedge k}\right)\right] .
$$

That is, $N$ is $(6 p+3)$-connected, and so we obtain

$$
\left[P^{4 p+4}(p), N\right]=0 \quad \text { and } \quad\left[P^{4 p+3}(p), N\right]=0 .
$$

From Proposition 2.1,

$$
\left[P^{4 p+3}(p), P^{4 p+3}(p)\right]=\mathbb{Z} / p \mathbb{Z}\{\iota\} \quad \text { and } \quad\left[P^{4 p+4}(p), P^{4 p+3}(p)\right]=0 .
$$

There is a commutative diagram, obtained from Diagram (1),

$$
\begin{aligned}
{\left[P^{4 p+3}(p), V^{4 p+3}\right] \longrightarrow } & {\left[P^{4 p+3}(p), P^{4 p+3}\right] \longrightarrow\left[P^{4 p+2}(p),(X, B)_{\infty}\right] } \\
\Sigma \uparrow & \cong \uparrow \text { incl. } \\
& {\left[P^{4 p+2}(p), P^{4 p+2}(p)\right] \stackrel{A_{*}}{\longrightarrow}\left[P^{4 p+2}(p), P^{2 p+4}(p)\right] }
\end{aligned}
$$

So the map $\left[P^{4 p+3}, V^{4 p+3}\right] \rightarrow\left[P^{4 p+3}(p), P^{4 p+3}(p)\right]$ is zero map. Therefore, the map induced by inclusion

$$
\left[P^{4 p+3}(p), P^{2 p+4}(p)\right] \longrightarrow\left[P^{4 p+3}(p), V^{4 p+3}\right]
$$

is bijective. From Proposition 2.1, $\left[P^{4 p+3}(p), P^{2 p+4}(p)\right]=0$ since it is stable. Hence $\left[P^{4 p+3}(p), V^{4 p+3}\right]=0$.

(ii) $i=6 p+2$. From Proposition 2.1,

$$
\left[P^{6 p+2}(p), P^{4 p+3}(p)\right]=0 \quad \text { and } \quad\left[P^{6 p+3}(p), P^{4 p+3}(p)\right]=0 .
$$

Since $N$ is $(6 p+3)$-connected, we obtain that

$$
\left[P^{6 p+2}(p), N\right]=0 \quad \text { and } \quad\left[P^{6 p+3}(p), N\right]=0 .
$$

Thus, $\left[P^{6 p+2}(p), F_{(X, B)}\right] \rightarrow\left[P^{6 p+2}(p), V^{4 p+3}\right]$ is bijective. Therefore, the map induced by inclusion

$$
\left[P^{6 p+2}(p), P^{2 p+4}(p)\right] \longrightarrow\left[P^{6 p+2}(p), V^{4 p+3}\right]
$$


Homology, Homotopy and Applications, vol. 8(1), 2006

is bijective. Now we will show $\left[P^{6 p+2}(p), P^{2 p+4}(p)\right]=0$.

From Proposition 2.6,

$$
\begin{aligned}
{\left[P^{6 p+2}(p), P^{2 p+4}(p)\right] \cong } & {\left[P^{6 p+1}(p), \Omega P^{2 p+4}(p)\right] } \\
\cong & {\left[P^{6 p+1}(p), S^{2 p+3}\{p\}\right] } \\
& \times\left[P^{6 p+1}(p), \Omega \Sigma \bigvee_{k \geqslant 0} P^{(4+2 k)(p+1)+2}(p)\right]
\end{aligned}
$$

By Corollary 2.3,

$$
\left[P^{6 p+1}(p), S^{2 p+3}\{p\}\right]=0 .
$$

By the Hilton-Milnor theorem,

$$
\Omega \Sigma \bigvee_{k \geqslant 0} P^{(4+2 k)(p+1)+2}(p) \simeq \Omega \Sigma\left(P^{4(p+1)+2}(p)\right) \times Y,
$$

where $\left[P^{6 p+1}(p), Y\right]=0$. The map

$$
E_{*}:\left[P^{6 p+1}(p), P^{4 p+6}(p)\right] \longrightarrow\left[P^{6 p+1}(p), \Omega \Sigma P^{4 p+6}(p)\right]
$$

induced by Freudenthal suspension is bijective from the Freudenthal suspension theorem. And by Proposition 2.1, $\left[P^{6 p+1}(p), P^{4 p+6}(p)\right]=0$ since it is stable. Hence $\left[P^{6 p+2}(p), P^{2 p+4}(p)\right]=0$.

\section{The unstable splitting of $V(1) \wedge V(1)$}

The purpose of this section is to prove Theorem 1.3. In order to prove this theorem, we use the following theorem of Cohen.

Theorem 5.1 (Cohen [1]). Assume that $X=\Sigma A$. If $X$ is a p-local space, then there exists a homotopy equivalence

$$
X \wedge X \longrightarrow(\underset{1-T}{\operatorname{hocolim}} X \wedge X) \vee(\underset{1+T}{\operatorname{hocolim}} X \wedge X)
$$

where $T: X \wedge X \longrightarrow X \wedge X$ is the transposition.

Denote $L_{2}(X)=\underset{1-T}{\operatorname{hocolim}} X \wedge X$ and $M_{2}(X)=\underset{1+T}{\operatorname{hocolim}} X \wedge X$. Since $V^{2 p+3}=$ $\Sigma V^{2 p+2}$, Theorem 5.1 says that

$$
V^{2 p+3} \wedge V^{2 p+3} \simeq L_{2}\left(V^{2 p+3}\right) \vee M_{2}\left(V^{2 p+3}\right) .
$$

Now we will compute the homology of $L_{2}\left(V^{2 p+3}\right)$ and $M_{2}\left(V^{2 p+3}\right)$.

Let $T^{-}=1-T$ and $T^{+}=1+T$. Since $T_{*}^{-} T_{*}^{-}=2 T_{*}^{-}$and $T_{*}^{+} T_{*}^{+}=2 T_{*}^{+}, p$ is an odd prime ( $\frac{1}{2}$ exists), and homotopy colimit commutes with homology,

$$
\begin{aligned}
H_{*}\left(L_{2}\left(V^{2 p+3}\right)\right) & \cong \underset{T_{*}^{-}}{\operatorname{colim}} H_{*}\left(V^{2 p+3} \wedge V^{2 p+3}\right) \\
& \cong \operatorname{Im} T_{*}^{-},
\end{aligned}
$$


and

$$
\begin{aligned}
H_{*}\left(M_{2}\left(V^{2 p+2}\right)\right) & \cong \operatorname{colim}_{T_{*}^{+}} H_{*}\left(V^{2 p+3} \wedge V^{2 p+3}\right) \\
& \cong \operatorname{Im}_{*}^{+}
\end{aligned}
$$

The reduced mod $p$ homology of $V^{n}, \widetilde{H}_{*}\left(V^{n}\right)$, is generated by $a, b, c$ and $d$ of degree $n, n-1, n-2 p+1$ and $n-2 p$, respectively, such that the homology Bockstein $\beta$ and $\mathcal{P}_{*}^{1}$ on them is

$$
\beta(a)=b, \quad \mathcal{P}_{*}^{1}(b)=c, \quad \beta(c)=d .
$$

We denote these relations by the following diagram:

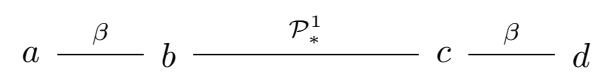

Then, $\widetilde{H}_{*}\left(V^{2 p+3}\right)$ is

$\begin{array}{cccc}2 p+3 & 2 p+2 & 4 & 3 \\ a & b & c & d .\end{array}$

Let us denote the generators of $\widetilde{H}_{*}\left(\Sigma\left(V^{2 p+2} \wedge V^{2 p+2}\right)\right)$ as follows:

$$
\begin{array}{llllllllll}
a a & a b & a c & a d & b b & b c & b d & c c & c d & d d \\
& b a & c a & d a & & c b & d b & & d c &
\end{array}
$$

where $a b$ means $a \otimes b \cdot \operatorname{Im} T_{*}^{-}$and $\operatorname{Im} T_{*}^{+}$are generated by the images of these generators under $T_{*}^{-}$and $T_{*}^{+}$, respectively. The following is immediate.

$$
\begin{aligned}
& T_{*}^{-}(a a)=2 a a, \quad T_{*}^{-}(b b)=0, \quad T_{*}^{-}(c c)=0, \quad T_{*}^{-}(d d)=2 d d, \\
& T_{*}^{-}(a b)=a b-b a=-T_{*}^{-}(b a), \\
& T_{*}^{-}(a c)=a c-c a=-T_{*}^{-}(c a), \\
& T_{*}^{-}(a d)=a d-d a=-T_{*}^{-}(d a), \\
& T_{*}^{-}(b c)=b c+c b=T_{*}^{-}(c b), \\
& T_{*}^{-}(b d)=b d-d b=-T_{*}^{-}(d b), \\
& T_{*}^{-}(c d)=c d-d c=-T_{*}^{-}(d c), \quad T_{*}^{+}(c c)=2 c c, \quad T_{*}^{+}(d d)=0, \\
& T_{*}^{+}(a a)=0, \quad T_{*}^{+}(b b)=2 b b, \\
& T_{*}^{+}(a b)=a b+b a=T_{*}^{+}(b a), \\
& T_{*}^{+}(a c)=a c+c a=T_{*}^{+}(c a), \\
& T_{*}^{+}(a d)=a d+d a=T_{*}^{+}(d a), \\
& T_{*}^{+}(b c)=b c-c b=-T_{*}^{+}(c b), \\
& T_{*}^{+}(b d)=b d+d b=T_{*}^{+}(d b), \\
& T_{*}^{+}(c d)=c d+d c=T_{*}^{+}(d c) .
\end{aligned}
$$


Homology, Homotopy and Applications, vol. 8(1), 2006

Their relations with $\beta$ and $\mathcal{P}_{*}^{1}$ are as follows:

$$
\begin{aligned}
\beta T_{*}^{-}(a a) & =2(b a-a b)=-2 T_{*}^{-}(a b), \\
\mathcal{P}_{*}^{1} T_{*}^{-}(a b) & =a c-c a=T_{*}^{-}(a c), \\
\beta T_{*}^{-}(a c) & =b c-a d-d a+c b=T_{*}^{-}(b c-a d), \\
\beta T_{*}^{-}(b c) & =-b d+d b=-T_{*}^{-}(b d), \\
\mathcal{P}_{*}^{1} T_{*}^{-}(b d) & =c d-d c=T_{*}^{-}(c d), \\
\beta T_{*}^{-}(c d) & =-2 d d=-T_{*}^{-}(d d), \\
\beta T_{*}^{+}(a b) & =2(b b)=T_{*}^{+}(b b), \\
\mathcal{P}_{*}^{1} T_{*}^{+}(b b) & =2(c b+b c)=2 T_{*}^{+}(b c), \\
\beta T_{*}^{+}(b c) & =-b d-d b=-T_{*}^{+}(b d), \\
\mathcal{P}_{*}^{1} T_{*}^{+}(a b) & =a c+c a=T_{*}^{+}(a c), \\
\beta T_{*}^{+}(a c) & =b c-a d+d a+c b=T_{*}^{+}(b c-a d), \\
\mathcal{P}_{*}^{1} T_{*}^{+}(b c-a d) & =2(c c)=T_{*}^{+}(c c), \\
\beta T_{*}^{+}(c c) & =2(d c+c d)=2 T_{*}^{+}(c d), \\
\mathcal{P}_{*}^{1} T_{*}^{+}(b c) & =2(c c)=T_{*}^{+}(c c), \\
\mathcal{P}_{*}^{1} T_{*}^{+}(b d) & =c d+d c=T_{*}^{*}(c d) .
\end{aligned}
$$

That is, if we replace generators of $\widetilde{H}_{*}\left(M_{2}\left(V^{2 p+2}\right)\right)$ and $\widetilde{H}_{*}\left(L_{2}\left(V^{2 p+2}\right)\right)$, they are represented as follows:

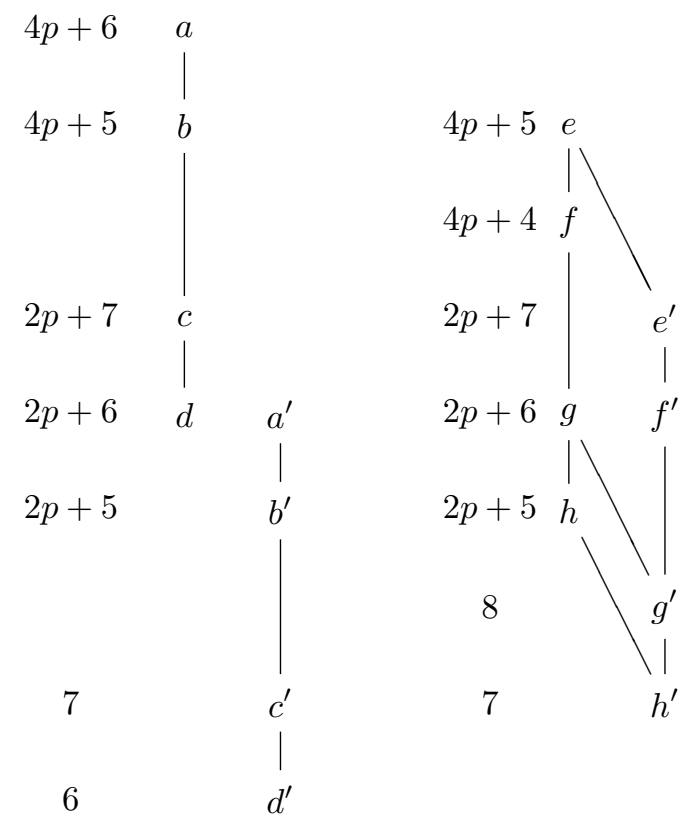

where the left-hand side diagram is for $\widetilde{H}_{*}\left(L_{2}\left(V^{2 p+3}\right)\right)$ and the right one is for 
$\widetilde{H}_{*}\left(M_{2}\left(V^{2 p+3}\right)\right)$.

Lemma 5.2. $\Sigma^{2 p-3} L_{2}\left(V^{2 p+3}\right) \simeq V^{6 p+3} \vee V^{4 p+3}$.

Proof. The elements of $\widetilde{H}_{*}\left(\Sigma^{2 p-4} L_{2}\left(V^{2 p+3}\right)\right)$ are connected by homology operations as follows:

$$
\begin{aligned}
& 6 p+26 p+1 \quad 4 p+3 \quad 4 p+24 p+1 \quad 2 p+32 p+2 \\
& a-b-c-d \\
& a^{\prime}-b^{\prime}-c^{\prime}-d^{\prime}
\end{aligned}
$$

There is a map $f: V^{4 p+2} \rightarrow \Sigma^{2 p-4} L_{2}\left(V^{2 p+3}\right)$ such that

$$
\begin{aligned}
& f_{*}\left(a_{4 p+2}\right)=a^{\prime}, \\
& f_{*}\left(b_{4 p+1}\right)=b^{\prime}, \\
& f_{*}\left(c_{2 p+3}\right)=c^{\prime}, \\
& f_{*}\left(d_{2 p+2}\right)=d^{\prime}
\end{aligned}
$$

for $f_{*}: H_{*}\left(V^{4 p+2}\right) \rightarrow H_{*}\left(\Sigma^{2 p-4} L_{2}\left(V^{2 p-3}\right)\right)$ because of $\operatorname{deg} a^{\prime}=4 p+2,4 p+2 \leqslant$ $\min \left\{2(2 p+1),(2 p+1)+2 p^{2}-2 p-3\right\}, \mathcal{P}_{*}^{1}\left(\mathrm{a}^{\prime}\right)=0$, and applying Theorem 1.1. Consider the cofiber sequence:

$$
V^{4 p+2} \stackrel{f}{\longrightarrow} \Sigma^{2 p-4} L_{2}\left(V^{2 p+3}\right) \longrightarrow C_{f} \longrightarrow V^{4 p+3} \longrightarrow \Sigma^{2 p-3} L_{2}\left(V^{2 p+3}\right)
$$

Since the $\bmod p$ homology of $C_{f}$ is isomorphic to that of $V^{6 p+2}$, Theorem 1.1 implies that there is a map from $V^{6 p+2}$ to $C_{f}$ which induces an isomorphism of $\bmod p$ homology. That is,$C_{f}$ is homotopy equivalent to $V^{6 p+2}$. Since, by Lemma $1.2,\left[V^{6 p+2}, V^{4 p+3}\right]=0$, we conclude $\Sigma^{2 p-3} L_{2}\left(V^{2 p+3}\right)=V^{6 p+3} \vee V^{4 p+3}$.

Proof of Theorem 1.3. Since $V^{m} \wedge V^{n}=\Sigma^{(m-2 p-3)+(n-2 p-3)} V^{2 p-3} \wedge V^{2 p-3}$ and $m+n-4 p-6 \geqslant 2 p-3$,

$$
\begin{aligned}
V^{m} \wedge V^{n} & \simeq \Sigma^{m+n-4 p-6}\left(L_{2}\left(V^{2 p+3}\right) \vee M_{2}\left(V^{2 p+3}\right)\right) \\
& \simeq\left(V^{m+n} \vee V^{m+n-2 p}\right) \vee \Sigma^{m+n-4 p-6} M_{2}\left(V^{2 p+3}\right) .
\end{aligned}
$$

The proof is complete by putting $M=\Sigma^{m+n-4 p-6} M_{2}\left(V^{2 p+3}\right)$.

\section{Samelson product}

In the previous section, we found a homotopy equivalence

$$
V^{m} \wedge V^{n} \longrightarrow V^{m+n} \vee V^{m+n-2 p} \vee M
$$

for $m, n \geqslant 2 p+3$ and $m+n \geqslant 6 p+3$. Let $\Delta_{m, n}: V^{m+n} \rightarrow V^{m} \wedge V^{n}$ be the composition of the inclusion and the homotopy inverse map. Let us define the Samelson product for $f: V^{m} \rightarrow \Omega X$ and $g: V^{n} \rightarrow \Omega X$ by

$$
[f, g]: V^{m+n} \stackrel{\Delta_{m, n}}{\longrightarrow} V^{m} \wedge V^{n} \stackrel{f \wedge g}{\longrightarrow} \Omega X \wedge \Omega X \stackrel{\langle,\rangle}{\longrightarrow} \Omega X .
$$

In the remainder of this section, we establish the properties claimed in Theorem 1.4 . 
Property (4) of Theorem 1.4: It is clear that

$$
h_{*}[f, g]=\left[h_{*} f, h_{*} g\right]
$$

for the H-map $h: \Omega X \rightarrow \Omega Y$ by the definition of $[f, g]$.

Property (5) of Theorem 1.4: According to Neisendorfer [7, Lemma 10.5], we obtain the following.

Lemma 6.1. If $x, y \in H_{*}(\Omega X)$ are primitive, then $[,]_{*}(x \times y)=[x, y]$, where $[$,$] :$ $\Omega X \times \Omega X \rightarrow \Omega X$ is commutator and $[x, y]=x y-(-1)^{|x||y|} y x$.

For all $f \in\left[V^{n}, X\right], \varphi(f)=f_{*}\left(a_{n}\right)$ is primitive because $a_{n} \in H_{n}\left(V^{n}\right)$ is primitive. If $a_{m}, a_{n}$ and $a_{m+n}$ denote the top cell of $V^{m}, V^{n}$ and $V^{m+n}$ respectively, then $\varphi\left(\Delta_{m, n}\right)=\left(\Delta_{m, n}\right)_{*}\left(a_{m+n}\right)=a_{m} \otimes a_{n}$. Hence, considering the following homotopy commutative diagram

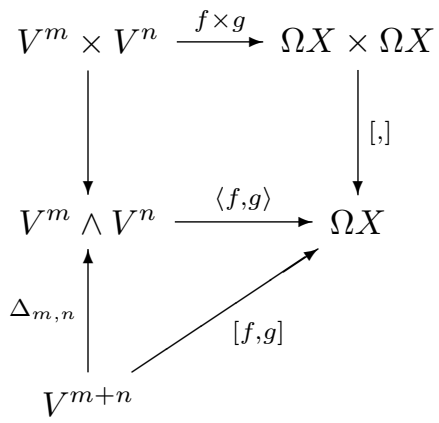

we obtain that

$$
\begin{aligned}
\varphi([f, g]) & =[f, g]_{*}\left(a_{m+n}\right)=\langle f, g\rangle_{*}\left(a_{m} \otimes a_{n}\right) \\
& =[,]_{*}(f \times g)_{*}\left(a_{m} \times a_{n}\right)=[,]_{*}(\varphi(f) \times \varphi(g)) \\
& =[\varphi(f), \varphi(g)],
\end{aligned}
$$

establishing Property (5).

Properties (1)-(3) of Theorem 1.4: To establish them, we recall the following proposition of Neisendorfer.

Proposition 6.2 ([7, Proposition 9.10]). Suppose $C_{1}, C_{2}$ and $C_{3}$ are coabelian (e.g. suspension), and suppose $G$ is a group-like space. Let $a, a^{\prime} \in\left[C_{1}, G\right]$, let $b, b^{\prime} \in$ $\left[C_{2}, G\right]$, and let $c \in\left[C_{3}, G\right]$. If we denote the $\langle a, b\rangle$ as the composition $C_{1} \wedge C_{2} \stackrel{a \wedge b}{\longrightarrow}$ $G \wedge G \stackrel{\langle,\rangle}{\longrightarrow} G$, then the following formulas hold:

(i) $\left\langle a+a^{\prime}, b\right\rangle=\langle a, b\rangle+\left\langle a^{\prime}, b\right\rangle$ and $\left\langle a, b+b^{\prime}\right\rangle=\langle a, b\rangle+\left\langle a, b^{\prime}\right\rangle$,

(ii) $\langle a, b\rangle=-\bar{T}_{(1,2)}^{*}\langle b, a\rangle$,

(iii) $\langle a,\langle b, c\rangle\rangle+\bar{T}_{(1,2,3)}^{*}\langle b,\langle c, a\rangle\rangle+\bar{T}_{(1,3,2)}^{*}\langle c,\langle a, b\rangle\rangle=0$,

where $\bar{T}_{(1,2,3)}$ denotes a transposition from $C_{1} \wedge C_{2} \wedge C_{3}$ to $C_{3} \wedge C_{1} \wedge C_{2}$. 
For $f: V^{m} \rightarrow \Omega X$ and $g: V^{n} \rightarrow \Omega X$, the Samelson product $[f, g]$ is a composition $\langle f, g\rangle \Delta_{m, n}$. Hence from the formula (i), we have the bilinearity

$$
\left[f+f^{\prime}, g\right]=[f, g]+\left[f^{\prime}, g\right] \text { and }\left[f, g+g^{\prime}\right]=[f, g]+\left[f, g^{\prime}\right]
$$

for $f, f^{\prime}: V^{m} \rightarrow \Omega X$ and $g, g^{\prime}: V^{n} \rightarrow \Omega X$, i.e. property (1) of Theorem 1.4 holds.

Suppose that the diagram

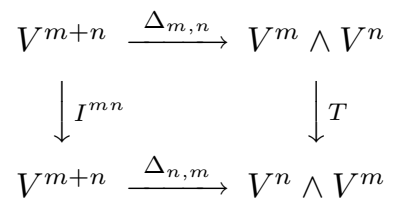

is commutative up to homotopy, where $I: V^{k} \rightarrow V^{k}$ is the map such that $I_{*}\left(a_{k}\right)=$ $-a_{k}$ in homology. Then formula (ii) of Proposition 6.2 implies anticommutativity (property (2) of Theorem 1.4).

Suppose that the diagram

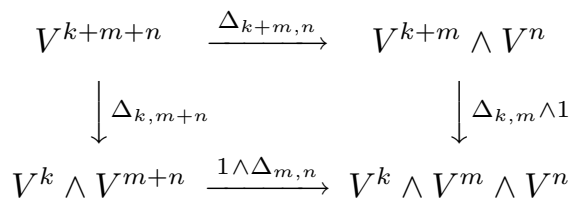

is commutative up to homotopy. Then formula (iii) of Proposition 6.2 implies the Jacobi identity (Property (3) of Theorem 1.4).

Proposition 6.3. Diagram (2) is commutative up to homotopy if $m+n \geqslant 8 p+2$. Moreover, property (2) of Theorem 1.4 holds.

Diagram (3) is commutative up to homotopy if $m+n \geqslant 12 p+2$. Moreover, property (3) of Theorem 1.4 holds.

Proof. Consider the element $a_{n} \otimes a_{m} \in H_{m+n}\left(V^{n} \wedge V^{m}\right)$. Since $m+n \geqslant 8 p+2$, $\mathcal{P}_{*}^{1}\left(a_{n} \otimes a_{m}\right)=0$ and $H_{m+n-2 p+3}\left(V^{n} \wedge V^{m}\right)=0$, there is a map $f: V^{m+n} \rightarrow V^{n} \wedge$ $V^{m}$ with $f_{*}\left(a_{m+n}\right)=(-1)^{m n} a_{n} \otimes a_{m}$ from Theorem 1.1. Moreover, such a map is unique up to homotopy because $H_{m+n-2 p+3}\left(V^{n} \wedge V^{m}\right)=0$. Since $\left(\Delta_{m, n}\right)_{*}\left(a_{m+n}\right)=$ $a_{m} \otimes a_{n}$, the top-right arrow and the left-bottom arrow in Diagram (2) satisfy this condition. The uniqueness implies that Diagram (2) is commutative up to homotopy.

Diagram (3) is also commutative because of the uniqueness of Theorem 1.1.

\section{References}

[1] F.R. Cohen, Fibration and product decompositions in nonstable homotopy theory. In Handbook of Algebraic Topology. North-Holland, Amsterdam, 1995, pp. $1175-1208$.

[2] F.R. Cohen, J.C. Moore, J.A. Neisendorfer, Torsion in homotopy groups. Ann. Math. 2 109(1) (1979), 121-168.

[3] F.R. Cohen and J.A. Neisendorfer, Note on desuspending the Adams map, Math. Proc. Cambridge Philos. Soc. 99(1) (1986), 59-64. 
[4] B. Gray, On the homotopy groups of mapping cones. Proc. London Math. Soc. 326 (1973), 497-520.

[5] B. Gray, EHP spectra and periodicity. I. Geometric constructions. Trans. Am. Math. Soc. 340(2) (1993), 595-616.

[6] J. Milnor, The Steenrod algebra and its dual, Ann. Math. 267 (1958), 150 171.

[7] J. Neisendorfer, Primary homotopy theory, Mem. Am. Math. Soc. 25(232) (1980), iv+67.

[8] H. Toda, Composition methods in homotopy groups of spheres. Annals of Mathematics Studies, 49. Princeton University Press, Princeton, NJ, 1962.

[9] H. Toda, Algebra of stable homotopy of $Z_{p}$-spaces and applications, J. Math. Kyoto Univ. 11 (1971), 197-251.

Takahisa Shiina shiina@math.shinshu-u.ac.jp

Department of Mathematical Sciences

Shinshu University

Matsumoto 390-8621

Japan

This article is available at http://intlpress.com/HHA/v8/n1/a5/ 